\title{
An Investigation of Drivers' Comprehension of Distracted Driving Slogans
}

\author{
Saidi Siuhi ${ }^{*}$, Judith Mwakalonge1, Rebecca Wright' ${ }^{1}$ Judy A. Perkins ${ }^{2}$ \\ ${ }^{1}$ Department of Engineering, South Carolina State University, Orangeburg, SC, USA \\ ${ }^{2}$ Civil and Environmental Engineering, Prairie View A\&M University, Prairie View, TX, USA \\ Email: ^ssiuhi@scsu.edu,jmwakalo@scsu.edu, rwrigh35@scsu.edu, juperkins@pvamu.edu
}

How to cite this paper: Siuhi, S., Mwakalonge, J., Wright, R. and Perkins, J.A. (2021) An Investigation of Drivers' Comprehension of Distracted Driving Slogans. Journal of Transportation Technologies, 11, 128-138. https://doi.org/10.4236/jtts.2021.112008

Received: August 4, 2020

Accepted: February 23, 2021

Published: February 26, 2021

Copyright ( 2021 by author(s) and Scientific Research Publishing Inc. This work is licensed under the Creative Commons Attribution International License (CC BY 4.0).

http://creativecommons.org/licenses/by/4.0/ (c) (i) Open Access

\begin{abstract}
Distracted driving occurs when a driver diverts the primary attention from driving to another task. Using mobile devices such as a cellphone for texting, calls, or other manipulation while driving has the highest potential for distraction because it combines both forms of distractions, manual, visual, and cognitive. Some states in the US have posted slogans including " $W 82 T X T$, it s a law", "Don't Drive inTEXTicated", "PLS dnt txt n drv", "Don't tempt F8 that txt can w8", and "DNT TXT \& DRV" along highways to convey the dangers and laws regarding distracted driving to minimize incidences of distracted-related crashes This study surveyed 347 people using the five distraction slogans in a college town. The results showed that younger drivers have a higher level of comprehension compared to older drivers. Further, the results showed that drivers with university education or more years of driving experience have a higher comprehension level of distraction signs compared to their counterparts.
\end{abstract}

\section{Keywords}

Distracted Driving, Road Safety Slogans, Road Sign Comprehension, Road Safety Campaign, Road Safety Awareness

\section{Introduction}

This research was born out of the personal experience when I first saw a distracted driving slogan "W8 $2 T x t$, It's a law" posted along Interstate 26 (I-26) in South Carolina. In this paper, a slogan is defined as a short and striking or memorable phrase displaying a distracting message to the traveling motorists or road users. Some of the posted slogans are regulatory sign intended to inform road users of selected laws or regulations and indicates the applicability of the 
legal requirements [1]. However, traffic signs are only effective when road users clearly understand and comprehend their meaning. Dewar [2] stated that the most important factor for a well-designed sign is the understandability of the sign. Additionally, Pline [3] pointed out for a traffic sign to be effective, it must satisfy the following requirements: fulfill a need, command attention, convey a clear and simple meaning, command the respect of the road users and give ample time for a proper response. Of these important requirements, the conveyance of a clear and simple meaning emphasizes the need for the understandability of road signs. Referencing a personal experience, the "W8 $2 T x t$, It's a law" message was not clear even after multiple sightings until after I conducted an online search for its meaning. Figure 1 shows a posted variable message on Interstate 5 at milepost 25 south of Medford, South Oregon, encouraging motorists to avoid distractions such as texting while driving [4]. The figure also shows a location of a sign on a highway and how it looks in a real driving environment.

Recognizing the need for understanding drivers' comprehension of traffic signs, numerous studies have been conducted on this topic. Early studies dating back to 1966 mainly focused on evaluating levels of users' understanding of local traffic signs. Most of the results indicated that general comprehension performance was far from satisfactory [5].

Yuan et al. [6] distributed a survey questionnaire to 550 drivers to test the perception accuracy of warning signs. Also, the study investigated age, gender, educational background, and years holding a license on drivers' comprehension of warning signs. The study found that age, gender, and driving years had no significant influence on sign comprehension. However, drivers with higher educational backgrounds presented better comprehension of warning signs than those with lower educational backgrounds [6].

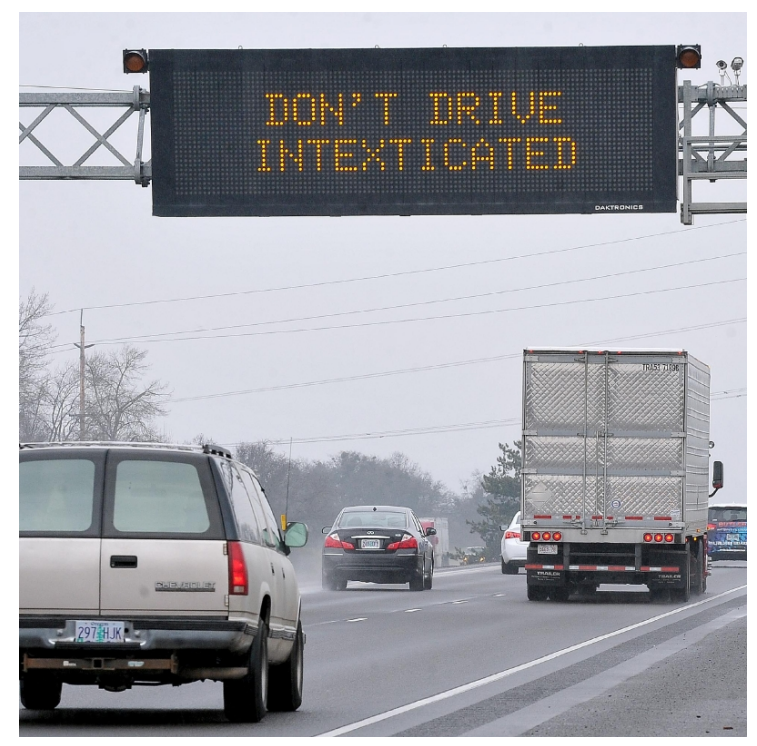

Figure 1. "Don't Drive inTEXTicated" sign posted on Interstate 5 at milepost 25 South of Medford, Southern Oregon [4]. 
Katz et al. [7] recruited 103 participants to investigate comprehension for several types of speed management signs including animal presence warnings involving a flashing beacon, regulatory and advisory warnings, variable speed limit signs with text, and animation. Participants viewed the signs, described what the signs meant, and then completed a comprehension task in which statements about each sign were presented and participants indicated whether the statement was true of the sign. The study found that: 1) many participants failed to recognize that a hazard may still exist when the beacon attached to the animal presence sign is off, 2) many people thought that an advisory speed on a warning sign is an enforceable legal speed limit, and 3) the variable speed limit sign was understood with either a text message or with animation.

Al-Madani and Al-Janahi [8] investigated the relationship between drivers' characteristics and their comprehension of posted signs. The characteristics considered in the study included experience, accident per experience ratio, age, marital status, sex type, nationality, educational background, and monthly income. The populations sampled were from Bahrain, Kuwait, Oman, Qatar, and the United Arab Emirates. A total of 28 posted signs were considered and investigated in the study. The study found that on average, drivers comprehended only $56 \%$ of the posted signs. Education, monthly income, and nationality were related to drivers' comprehension of traffic signs. Male drivers scored higher than female drivers. Also, the study found that age, marital status, experience, and accident rates did not affect drivers' comprehension of signs.

Zhang and Chan [9] reviewed both recent and early studies concerning traffic sign comprehension and found that unsatisfactory comprehension was a common problem for drivers in many countries. Additionally, the study found that most studies showed a positive relation between comprehension level and educational background. However, the effects of other factors on comprehension levels were not unanimous.

Klauer et al. [10] reported that drivers who are 15 to 20 years of age constituted $6.4 \%$ of all drivers, but they account for $10.0 \%$ of all motor vehicle traffic deaths and $14.0 \%$ of all police-reported crashes resulting in injuries. These rates are thought to result from a combination of young age, inexperience, and risky driving behaviors [10].

Many factors that cause and/or contribute to traffic crashes are related to the vehicle, infrastructure, and human. Several human factors such as distracted driving, over speeding, fatigue driving, drunk driving, and overloading/overcrowding driving are the primary traffic crashes causes [11]. Distracted driving is dangerous and claimed 2841 lives in 2018 alone. Among those killed were 1730 drivers, 605 passengers, 400 pedestrians, and 77 bicyclists [12]. Figure 2 shows distractedaffected fatalities and cell phone use fatalities trends from 2014 to 2018 [12]. The most cited distracting activities include talking on, listening to, or manipulating a cellphone (or other cellphone activity) at the time of the crash. Even though the trends are showing a decreasing fatality over time, NHTSA is dedicated to eliminating distracted-affected risky behaviors on our nation's roads. 


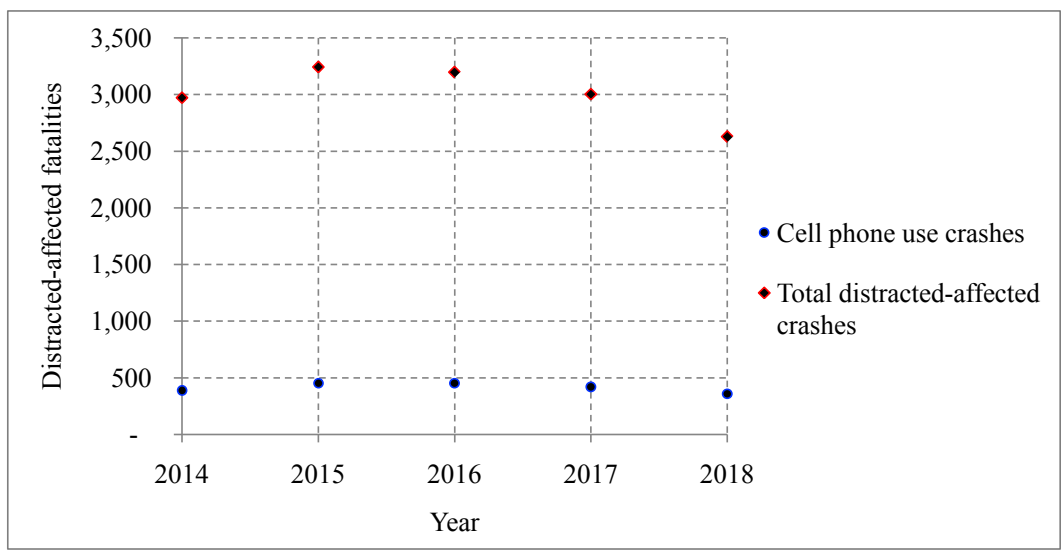

Figure 2. Distracted-affected fatalities, 2014-2018 [12].

To eliminate distracted driving behavior, the NHTSA is leading the national effort to save lives by preventing this dangerous behavior. It encourages all road users to get the facts, get involved, and help NHTSA keep America's roads safe. In similar efforts to reduce the negative impacts of distracted-affected crashes, transportation agencies have started to post variable message signs to warn drivers about the consequences of distracted driving. Some of the signs posted, however, drivers may have difficulty comprehending and understanding the meaning.

Therefore, the objective of this paper is to determine the comprehension levels of road users on some of the posted distracted driving slogans. It looks at the impact of gender, age, education, and years of driving license on comprehension levels of the posted distracted driving slogans. The following section presents the methods used to achieve study objectives, results, and discussions, a summary of key findings, conclusions, and recommendations for future research directions.

\section{Methodology}

This paper used a survey questionnaire approach to document the comprehension levels of different individuals. The survey was disseminated and completed by participants in a college town, Orangeburg, South Carolina. Several distracting slogans exist in the literature, however, based on the review, five slogans were selected for further investigation. It is worthwhile mentioning that different states have implemented different slogans to address the distracted driving problems in their state and/or local jurisdictions. Thus, the selected slogans may not capture all slogans, but they provide an initial insight on what should be done in terms of design to improve their comprehension performance. The sub-sections that follow provide a detailed description of the selected slogans, survey sites, and questionnaire contents.

\subsection{Slogan Selection}

Slogan selection involved the identification of slogans to be used in the survey. 
Two criteria were set for the selection of slogans. First, it should be a distracted driving slogan that is utilized by one or more transportation agencies or safety advocates in the United States. Second, their message is conveyed with acronyms and/or numbers, because these were perceived as not simple to understand. Table 1 presents the selected slogans along with their basic information. All selected slogans used acronyms and only two slogans had both acronyms and numbers.

\subsection{Survey Sites}

The research sites consisted of three post-secondary institutions in Orangeburg, South Carolina, a city of 13,964 people as of the 2010 census (America Factfinder, 2013). The three institutions were South Carolina State University (SC State), a public university of about 2500 - 3000 students, Claflin University, a private university of about 1800 - 2000 students, Orangeburg-Calhoun Technical College, the area community college of about 2500 . This survey was issued to students, faculty, and staff of these institutions. The survey was conducted between the last week of June and the first week of July 2015.

\subsection{Questionnaire Design and Contents}

The survey questionnaire included an introduction explaining the purpose of the study and two pages of questions as shown in Figure 3. Following the introduction, the questionnaire consisted of two parts. Part A was composed of the five slogans and participants were required to choose whether they understood the slogan or not. If the participant responded yes to a slogan, then they were required to write the meaning. Part B of the questionnaire consisted of four questions designed to capture drivers' characteristics, including gender, age, years of holding a driver's license, and education level (see Figure 3). Self-identifying information, such as name, address, and date of birth was not collected. In total, 347 surveys were completed and maintained in a Microsoft Excel spreadsheet.

\section{Results and Discussions}

This section presents the results of the survey responses obtained and discusses in detail their implications in understanding distracted driving slogans comprehension performance. The results include demographic characteristics of respondents and the percentage of messages/slogans correctly identified by respondents.

\subsection{Basic Information of Participants}

Table 2 presents the participants' responses to each of the Part B questions. More than $66 \%$ of all respondents were male and those aged 16 - 25 represented $50 \%$ of the respondents (expected because of the college setting). About $71 \%$ amongst the participants had a minimum of a university education level and the majority (63\%) had at least four years of holding a driving license. 
Table 1. Slogans' basic information.

\begin{tabular}{cccc}
\hline Sign code & How it reads & Meaning & States \\
\hline A & W8 2 TXT, it's a law & Wait to text, it's a law & South Carolina, Nebraska \\
B & Don't Drive inTEXTicated & Don't drive and text & Oregon, Missouri \\
C & PLS dnt txt $n d r v$ & Please don't text and drive & Cleveland, Ohio \\
D & Don't tempt F8 & Don't tempt fate & New Jersey, Pennsylvania \\
E & that txt can w8 & that text can wait & California, New York
\end{tabular}

Table 2. Participants' basic information.

\begin{tabular}{cccc}
\hline Driver factor & Response & Number & Percentage (\%) \\
\hline \multirow{2}{*}{ Gender } & Male & 115 & 33.2 \\
& Female & 231 & 66.8 \\
\multirow{3}{*}{ Age } & $16-25$ & 174 & 50.1 \\
& $26-50$ & 93 & 26.8 \\
Education Level & Over 50 & 80 & 23.1 \\
& Below University & 98 & 28.7 \\
& University or above & 244 & 71.3 \\
& Less than 2 years & 93 & 27.6 \\
Years of license & 2 to less than 4 years & 30 & 8.9 \\
& At least 4 years & 213 & 63.2 \\
& 4 & 1 & 0.3 \\
\hline
\end{tabular}

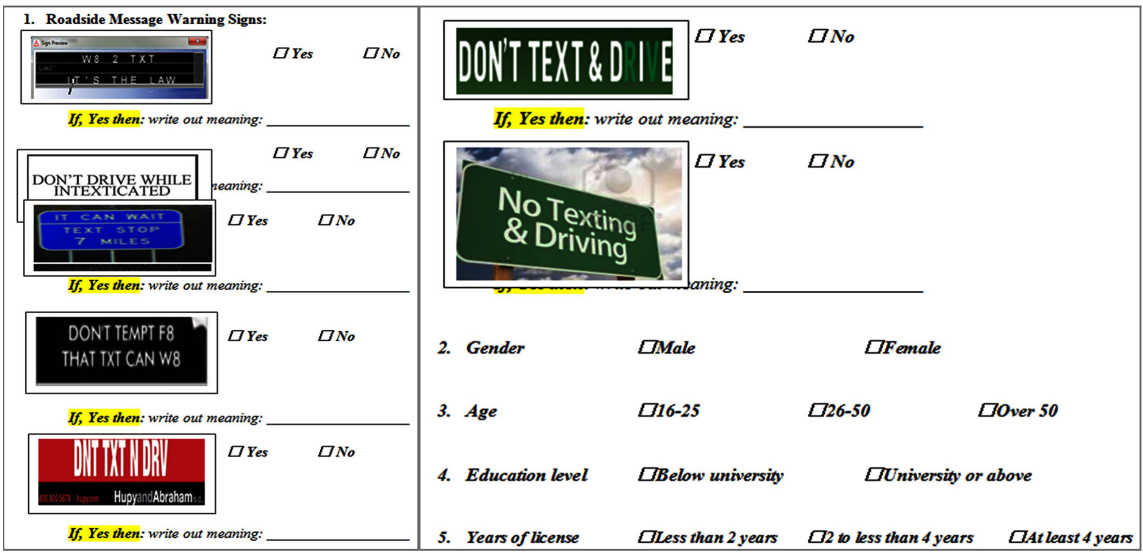

Figure 3. Content of survey questionnaire.

\subsection{Slogan Comprehension Score}

According to International Organization for Standardization (ISO) 3846 (2002), signs are considered acceptable when it scores at least $67 \%$ accuracy in a comprehension test [13]. The criterion set by the American National Standard Institute (ANSI) requires a sign to achieve a comprehension score of at least $85 \%$ accuracy. A comprehension score is computed as:

$$
\text { Comprehension score }(\%)=\frac{\text { number of slogan spelled correctly }}{\text { total number of responses }} \times 100
$$


According to ANSI, of the five slogans, only two passed the $85 \%$ accuracy criterion as shown in Table 3. The least comprehended sign was "Don't tempt F8 that txt can w8" which had a comprehension score of 55\%. As for South Carolina, more than $20 \%$ of the respondents did not understand the "W8 2 TXT, It's a LaW" a majority of whom were 50 years or older (32 out of 75 represent more than $42 \%)$. Similar results were reported by Richards and Heathington [14] found that elderly drivers (over 54 years) had difficulty in the comprehension of traffic signs.

Figure 4 displays a summary of responses from the respondents who completed the survey questionnaire. Looking at gender as shown in Figure 4, female drivers consistently displayed higher comprehension levels compared to males. This finding contradicts past studies conducted in Asia [6] [8]. Zhang and Chan [9] suggested that this finding could be due to the congenital difference in space perception or intellectual functioning or different cultures and exposure rates to signs between males and females. This is the subject of further investigation. On the other side, both male and female drivers showed a low comprehension score for the slogan "D Don't tempt F8 that txt can $w 8$ ".

Table 3. Percentage of the drivers' comprehending slogan message correctly.

\begin{tabular}{ccccccc}
\hline $\begin{array}{l}\text { Sign } \\
\text { code }\end{array}$ & How it reads & Correct & Wrong & $\begin{array}{c}\text { Comprehension } \\
\text { score (\%) }\end{array}$ & $\begin{array}{c}\text { 67\% ISO } \\
\text { accuracy }\end{array}$ & $\begin{array}{c}\text { 85\% ANSI } \\
\text { accuracy }\end{array}$ \\
\hline A & W8 2 TXT, it's a law & 272 & 75 & 78.39 & Pass & Fail \\
B & Don't Drive inTEXTicated & 229 & 111 & 67.36 & Pass & Fail \\
C & PLS dnt txt n drV & 299 & 45 & 86.92 & Pass & Fail \\
D & Don't tempt F8 that txt can w8 & 185 & 148 & 55.56 & Fail & Fail \\
E & DNT TXT \& DRV & 285 & 36 & 88.79 & Pass & Pass \\
\hline
\end{tabular}

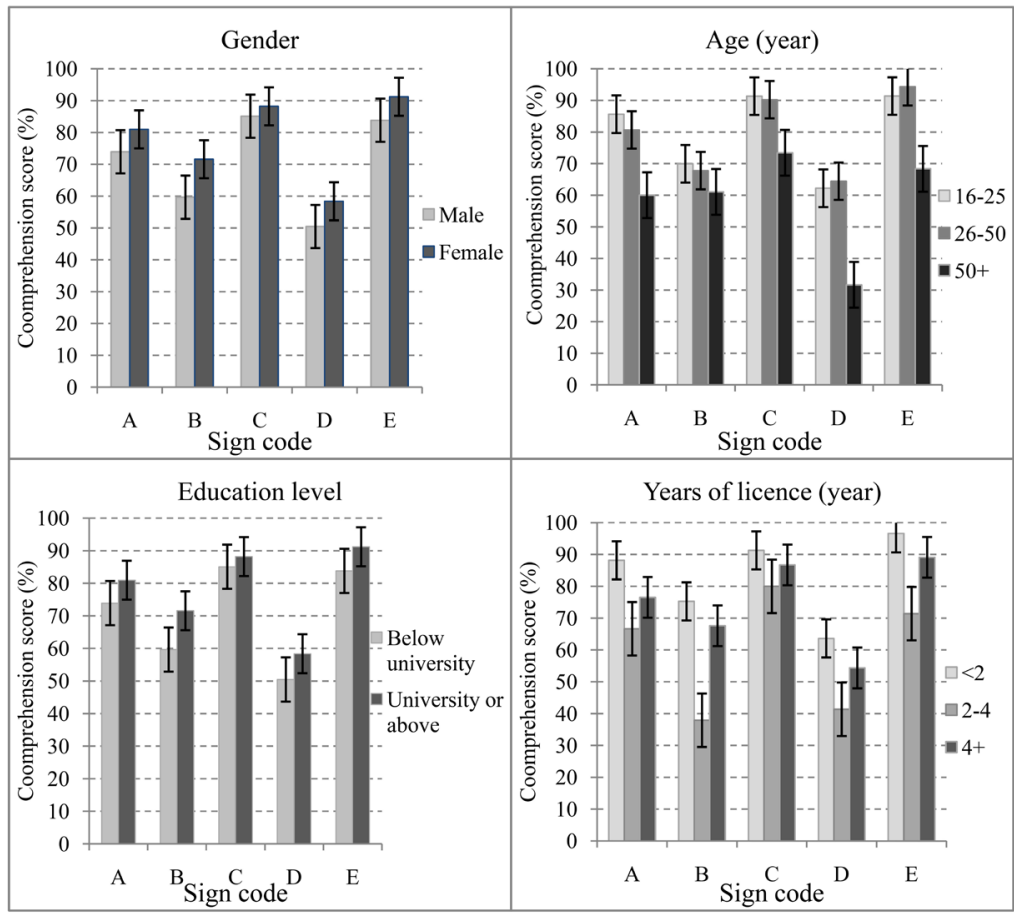

Figure 4. Drivers' response by personal characteristics. 
For the age factor, the comprehension level difference between 16 - 25 and 26 - 50 age groups did not exceed 3\% whereas the comprehension level difference between 26 - 50 and over 50 age groups exceeded 20\%. Older drivers (over 50 years) had the lowest comprehension scores. On average, the comprehension level decreases with age. Similar results are reported in (Dewar, 1988) who found that approximately $39 \%$ of US traffic signs were better understood by young drivers both before and after modifications to some of the symbols. Likewise, past research found that younger drivers possessed much more traffic safety knowledge than older drivers [15]. A possible explanation would be generally because young people have better information processing capabilities as well as a better vision than older drivers [16]. Other studies [6] [14] showed that middle-aged drivers had a higher comprehension level compared to other groups. However, both studies still showed that older drivers had difficulty in the comprehension performance of signs. Consistent with other studies [6] [8] [17], drivers with university education have a better comprehension of distracted driving slogans compared to their counterparts. However, among drivers with a university education, only two slogans (slogan "C"- "PLS dnt txt ndrv" and "E"-"DNT $\left.T X T \& D R V^{\prime \prime}\right)$ out of five met the ANSI 85\% accuracy criterion.

For the driving experience factor, this research used the Simpson [18] definition where driving experience is taken as the number of years licensed to drive. Interestingly, drivers with less than two years of holding a driving license have higher comprehension scores on the posted distracted driving slogans compared to other drivers. Further analysis reveals that the majority of those with less than two years of holding a driving license fall in the $16-25$ age group showed a higher comprehension level compared to other age groups. This is not surprising because young people usually have better information processing than older people.

\section{Summary and Conclusions}

This paper surveyed college students, faculty, and staff to determine the comprehension level of road users on the posted distracted driving slogans. The paper investigated five posted distracted driving messages along roadways in different states including "W8 $2 T X T$, it's a law", "Don't Drive inTEXTicated", "PLS dnt txt n drv", "Don't tempt F8 that txt can w8", and "DNT TXT \& DRV". A survey questionnaire was designed and distributed among the university population to document their understanding of the posted slogans and 347 participants responded to the questionnaire. Results showed that more than $10 \%$ of the participants did not have a clear understanding of at least one or more distracted driving messages displayed along highways e.g. "W8 2 TXT, It's a LaW". In summary, the study found the key findings:

1) Overall, the results showed that all participants had difficulty understanding the slogan "Don't tempt F8 that txt can w8." On the other side, slogan messages with simple acronyms "DNT TXT \& DRV" and "PLS dnt txt $n d r v$ " showed 
higher comprehension scores compared to other slogans. This finding further emphasizes the importance of providing simple messages while commanding drivers' attention.

2) Education plays an important role in drivers' ability to understand and comprehend driving distraction slogans. Participants with a university education have higher comprehension levels relative to those without a university education. Therefore, educational and awareness programs should focus more attention on drivers with lower educational backgrounds.

3) It was also found that age affects comprehension levels of distraction slogans. Younger drivers had a higher comprehension level compared to older drivers. With the increase in older populations, this may pose a challenge in achieving the ANSI 85\% accuracy criterion for posting a regulator sign along the roadways.

In conclusion, pilot programs for message designs should be investigated before their implementation to increase their effectiveness. The agency can reach out and invite the public through online, social media, or mail to get their opinions of the proposed distracted driving slogans for implementation. This will assist the agencies to select the slogan that conveys a simple message, easy to read and comprehend for implementation to reduce the incidences of distracted driving. The latter approach was utilized by the Tennessee Department of Transportation (TDOT) [19] that held an online contest asking people to submit safety messages they wanted to see displayed on the state's dynamic message signs throughout the upcoming year. Thereafter the TDOT selected the top five messages.

This paper had several limitations that need further research to address them. The following is a list of some shortcomings and opportunities for conducting future research in this area of distracted driving road signs to better communicate with travelers so that they can clearly understand and comprehend the meaning easily.

1) The paper used a college setting for its survey; thus, findings may not apply to the general public. The survey results, however, at least provided some highlights and insights on the relevance of this research. For more generalized results it is appropriate to survey other drivers and/or road users. Besides, all respondents were residents of South Carolina who were likely to be familiar with the "W8 2 TXT, it's a law" message. This may have introduced biases in the results and may not be transferable to other states or regions.

2) In this paper, no statistical inferences were drawn, thus, there is a need for future research works to address this limitation.

3) Personal characteristics considered in this study were limited to age, gender, education, and driving experience, however, other factors like marital status, occupation, cultural background, and driving practices may affect drivers' ability to understand and comprehend posted distracted driving traffic signs.

4) The current research focused on five posted distracted driving slogans. In 
future research, we will investigate more posted distracted driving slogans along roadways in the US.

\section{Conflicts of Interest}

The authors declare no conflicts of interest regarding the publication of this paper.

\section{References}

[1] Federal Highway Administration (FHWA) (2009) Manual on Uniform Traffic Control Devices for Street and Highways. US Department of Transportation, Washington DC.

[2] Dewar, R. (1988) Criteria for the Design and Evaluation of Traffic Sign Symbols. Transportation Research Record, 1160, 1-6.

[3] Pline, J.L. (1992) Traffic Engineering Handbook. Institute of Transportation Engineers, Prentice-Hall, Upper Saddle River.

[4] Freeman, M. (2017) Pay Attention! Mail Tribune. https://mailtribune.com/news/happening-now/pay-attention

[5] Johansson, G. and Rumar, K.R. (1966) Drivers and Road Signs: A Preliminary Investigation of the Capacity of Car Drivers to Get Information from Road Signs. Ergonomics, 9, 57-62. https://doi.org/10.1080/00140136608964342

[6] Yuan, L., Ma, Y. and Xu, P. (2014) Driver's Comprehension and Improvement of Warning Signs. Advances in Mechanical Engineering, 6, 1-9. https://doi.org/10.1155/2014/582606

[7] Katz, B., Rousseau, G. and Warren, D. (2003) Comprehension of Warning and Regulatory Signs for Speed. Annual Meeting Proceedings, Seattle, 24-27 August 2003.

[8] Al-Madani, H. and Al-Janahi, A.R. (2002) Assessment of Drivers' Comprehension of Traffic Signs Based on Their Traffic, Personal and Social Characteristics. Transportation Research Part F: Traffic Psychology and Behaviour, 5, 363-376. https://doi.org/10.1016/S1369-8478(02)00006-2

[9] Zhang, T. and Chan, A.H.S. (2013) Traffic Sign Comprehension: A Review of Influential Factors and Future Directions for Research. Proceeding of the International Multi-Conference of Engineers and Computer Scientists, Hong Kong, 13-15 March 2013, 2.

[10] Klauer, S.G., Guo, F., Simons-Morton, B.G., Marie Claude Ouimet, M.P.H., Lee, S.E. and Dingus, T.A. (2013) Distracted Driving and Risk of Road Crashes among Novice and Experienced Drivers. The New England Journal of Medicine, 370, 54-59. https://doi.org/10.1056/NEJMsa1204142

[11] World Health Organization (WHO) (2013) Global Status Report on Road Safety 2013: Supporting a Decade of Action. Violence and Injury Prevention. World Health Organization, Geneva.

[12] National Highway Traffic Safety Administration (NHTSA) (2020) Distracted Driving. https://www.nhtsa.gov/risky-driving/distracted-driving

[13] International Standards Organization (ISO) (2002) Graphical Symbols-Safety Colours and Safety Signs, Part 1: Design Principles for Safety Signs in Workplaces and Public Areas, Switzerland.

[14] Richards, S.H. and Heatherington, K.W. (1988) Motorist Understanding of Railroad-Highway Grade Crossing Traffic Control Devices and Associated Traffic Laws. 
Transportation Research Record, 1160, 52-59.

[15] International Road Federation (IRF) (1998) 3rd IRF Middle East Regional Meeting: Proceedings; towards Better Road Performance. Traffic and Safety. International Road Federation (IRF), 2.

[16] Vallesi, A., Stuss, D.T., McIntosh, A.R. and Picton, T.W. (2009) Age-Related Differences in Processing Irrelevant Information: Evidence from Event-Related Potentials. Neuropsychologia, 47, 577-586.

https://doi.org/10.1016/j.neuropsychologia.2008.10.018

[17] Ng, A.W.Y. and Chan, A.H.S. (2008) The Effects of Driver Factors and Sign Design Features on the Comprehensibility of Traffic Signs. Journal of Safety Research, 39, 321-328. https://doi.org/10.1016/j.jsr.2008.02.031

[18] Simpson, H.M. (2003) The Evolution and Effectiveness of Graduated Licensing. Journal of Safety Research, 34, 25-34. https://doi.org/10.1016/S0022-4375(02)00077-4

[19] Gordon, D. (2015) Tennessee Department of Transportation Online Contest for Road Messaging Signs.

https://www.davidgordonlaw.com/blog/tennessee-dept-of-transportation-online-co ntest-for-road-messaging-signs/ 